\title{
Negative contrast as a function of the location of small reinforced placements
}

\author{
RICHARD S. CALEF, EARL McHEWITT, DONALD W. MURRAY, \\ JAMES R. BROGAN, and RICHARD D. CAMERON \\ West Virginia Wesleyan College, Buckhannon, West Virginia 26201 \\ and \\ E. SCOTT GELLER \\ Virginia Polytechnic Institute and State University, Blacksburg, Virginia 24061
}

\begin{abstract}
After receiving large rewards in a straight gray runway during Phase 1, rats were given small rewards during placement trials (Phase 2) in a gray housing cage or in a gray goalbox. During Phase 3, only animals receiving small reward in housing cage placement trials showed a successive negative contrast effect (NCE). The results suggest that alley cues other than brightness mediate the NCE and are supportive of frustration theory.
\end{abstract}

Animal research in instrumental conditioning is replete with data showing the effects of a shift in reward magnitude (Black, 1968) in the runway. Specifically, many studies have shown that rats shifted from large to small magnitude of reward run more slowly than rats maintained on small magnitude of reward, a phenomenon designated as the negative contrast effect (NCE) (e.g., Capaldi \& Lynch, 1967; Crespi, 1942;Ehrenfreund, 1971; Gonzales, Gleitman, \& Bitterman, 1962). Studies by Calef and his associates (Calef, 1972; Calef, Calef, Prochaska, \& Geller, 1978; Calef, Hopkins, McHewitt, \& Maxwell, 1973; Maxwell, Calef, Murray, Shepard, \& Norville, 1976) have shown the frustration theory account of the NCE (Amsel, 1958, 1962) to be the most reliable. Frustration theory suggests that alley cues are particularly important in producing the NCE. According to Amsel $(1958,1962)$, alley cues such as brightness should elicit the "expectancy" (rg-sg) for large reward during the initial postshift period. After receiving less than "expected," the subjects should experience frustration and produce depressed speeds.

Recently, Capaldi (1978) presented results that appear to conflict with a frustration interpretation of the NCE ("depression" effect). In the Capaldi study, animals that received a simultaneous shift in reward and alley brightness (large black to small white) produced approximately as much of a NCE as animals that received only a shift in reward (large white to small white). These results imply that alley cues (brightness) were not instrumental in mediating a NCE. In other words, if alley cues were playing a major role in mediating the "depression" effect, changing the brightness from one phase to another should have resulted in less rg-sg to large reward being elicited by the alley, which in turn

Reprint requests should be sent to Richard S. Calef, West Virginia Wesleyan College, Buckhannon, West Virginia 26201. should have produced less frustration and depressed speeds.

However, Capaldi's (1978) findings may be supportive of a frustration account of the "depression" effect in that brightness may not be the main alley cue playing a significant role in mediating a NCE. Other cues such as texture, size, shape, or the cover of the apparatus may have been the relevant cues associated with large reward expectancy, since these cues did not change from preshift to postshift periods. To eliminate the latter cues would necessitate presenting the postshift phase in an apparatus other than the runway. This, of course, would make the findings less applicable to previous NCE research produced in the runway, since using a different apparatus would require subjects to make a response topographically different from running (e.g., leverpressing).

The purpose of the present study was to investigate whether cues other than brightness (e.g., texture, size, shape, or cover of runway apparatus) may have been the relevant cues mediating a NCE in the Capaldi (1978) study. Thus, the present study investigated the importance of alley cues other than brightness in producing the NCE while maintaining the postshift phase and searching for the "depression" effect in the runway. Two groups of experimental rats were given large (alley running trials), small (placement trials), and small (alley running trials) magnitudes of reward during three phases of the experiment, respectively. One group was given second-phase noncontingent reinforcement during goalbox (gray) placement trials, whereas the other group was given second-phase noncontingent reward during home cage (gray) placement trials. Two control groups were given small rewards throughout the study.

Since previous studies (Capaldi \& Lynch, 1967; Crespi, 1942; Ehrenfreund, 1971; Gonzales et al., 1962) have shown the NCE to be a transient phenomenon, as 
predicted by Amsel's (1958, 1962) frustration theory, it was hypothesized that only those rats given Phase 2 placement trials in the home cage would show a NCE during Phase 3 if alley cues other than brightness were mediating the NCE. According to frustration theory, alley cues should play a dominant role in the present study and elicit an expectancy (rg-sg) of large reward during the terminal Phase 1 period for both experimental groups. In the initial stages of Phase 2, some animals received small reward placement trials in the gray goalbox, which contained cues (brightness, texture, size, shape, and cover) similar to those of the alley. Theoretically, this should elicit rg-sg for large reward. As a consequence of continuing to receive small reward during this time, the subjects should experience frustration (rf-sf) that dissipates by the end of Phase 2.

During initial Phase 3, the alley cues should elicit anticipation of small reward (developed in Phase 2), and no NCE should be obtained, since the animals received what they anticipated. Animals that received small reward placement trials in the gray home cage, which did not contain as many similar cues to that of the alley, should experience neither a significant amount of anticipation of large reward nor frustration during this phase. During initial Phase 3, the alley cues should elicit anticipation of large reward, which should not be modified during Phase 2 . The downward shift in incentive should produce a NCE due to conditioned interfering responses elicited by frustration stimuli (rf-sf). It should be emphasized that if brightness is the relevant cue mediating the NCE, then neither placement group should show a NCE during Stage 3 , since neither group received a brightness change during placement trials. On the other hand, if brightness is not the relevant cue, then only those subjects that received placements in the home cage should show a NCE in Stage 3, since this is the only group that received a change in many other cues related to the runway.

\section{METHOD}

\section{Subjects}

The subjects were 32 experimentally naive male albino rats of the Sprague-Dawley strain, approximately 90 days old at the beginning of the experiment. The animals were acquired from Flow Laboratories, Dublin, Virginia, where they had been bred and raised.

\section{Apparatus}

The runway apparatus was essentially the same as that used by Ludvigson and Gay (1966), except that only one of the multiple parallel alleys was used. The alley was composed of a $33.02-\mathrm{cm}$ gray startbox, a $66.04-\mathrm{cm}$ gray runway section, and a $33.48-\mathrm{cm}$ gray goalbox. The inner width and height of each section of the runway were $7.62 \mathrm{~cm}$. Photocell and clock circuitry provided an independent measure of traversal time over the last $45.72-\mathrm{cm}$ segment of the run section. The apparatus contained a gray retrace door, separating the goal from the alley section, and an opaque Plexiglas ceiling.

\section{Procedure}

Fourteen days prior to the 1st day of training (Day 15), all rats were placed on a $23-\mathrm{h}$ food-deprivation schedule, which was maintained throughout the study. On Days $7-14$, the rats were taken from their individual home cages and handled for about 2 min each. On Days 13 and 14, the rats were taken to the experimental laboratory, where they were allowed to explore start and run sections of the apparatus while the clocks, photocells, and doors were operated. On those days, approximately $1 \mathrm{~g}$ of $45-\mathrm{mg}$ Noyes pellets, indentical to the subsequent reinforcement pellets, was incorporated into the rats' daily food ration.

Eight rats were randomly assigned to each of four groups. During Phase 1 , both experimental groups received 45 trials of large reward (12 pellets) following a traversal in a straight gray runway, whereas the two control groups received small reward (1 pellet) after a run. During Phase 2, in which all four groups received a total of 20 placement trials in 1 day, Group $L_{\mathrm{hc}} \mathrm{S}$ received small reward in a gray plastic housing cage (all of the rats in the present study were housed in wire mesh cages), and Group $\mathrm{LS}_{\mathrm{gb}} \mathrm{S}$ received small reward in a gray goalbox. The two remaining control groups, $\mathrm{SS}_{\mathrm{hc}} \mathrm{S}$ and $\mathrm{SS}_{\mathrm{gb}} \mathrm{S}$, received small reward throughout the second phase, in the home cage and in the goalbox, respectively. During Phase 3, small reward was given to all groups following a traversal down the gray runway for 35 trials.

All groups received a graduated increase in trials (i.e., one trial on the 1st day of training, two trials on the 2 nd day, etc.) and five trials on every day beginning from Day 20 . Trials were administered to four squads of eight rats each consisting of two animals from each group. The running order of subjects within a squad was randomized from day to day. The intertrial interval was approximately $10 \mathrm{~min}$, since a subject did not receive its second daily trial until all subjects in the squad had completed the first trial. The running trials during Phases 1 and 3 were initiated by placing the rat in the startbox. After a 3-sec orientation period, the start door was opened, and the rat was allowed to traverse the runway. Following the rat's entry into the goalbox, the retrace door was dropped. The rat was allowed to consume the pellet and was then removed from the apparatus to a nearby wire-mesh cage to await its next trial.

\section{RESULTS}

\section{Phase 1: Preshift Training}

Run times were converted to reciprocals, yielding run speeds. Figure 1 illustrates group mean speeds as a function of five-trial blocks. As can be seen in Figure 1, subjects receiving large magnitude of reward in Phase 1 (Groups $\mathrm{LS}_{\mathrm{hc}} \mathrm{S}$ and $\mathrm{LS}_{\mathrm{gb}} \mathrm{S}$ ) demonstrated faster running speed than small-reward control rats (Groups $\mathrm{SS}_{\mathrm{hc}} \mathrm{S}$ and $\mathrm{SS}_{\mathrm{gb}} \mathrm{S}$ ). A treatment (large and small reward) by location (home cage vs. goalbox) analysis of variance was performed on the data from Block 9. The analysis yielded a significant effect of treatment $[F(1,28)=3.89$, $\mathrm{p}<.05]$. None of the other effects reached significance $(\mathrm{p}>.05)$.

\section{Phase 3: Postshift (NCE)}

As can be seen in Figure 1, only the experimental group receiving its placement trials in the home cage (Group $\mathrm{LS}_{\mathrm{hc}} \mathrm{S}$ ) during Phase 2 showed significantly slower speeds relative to that of its respective control 


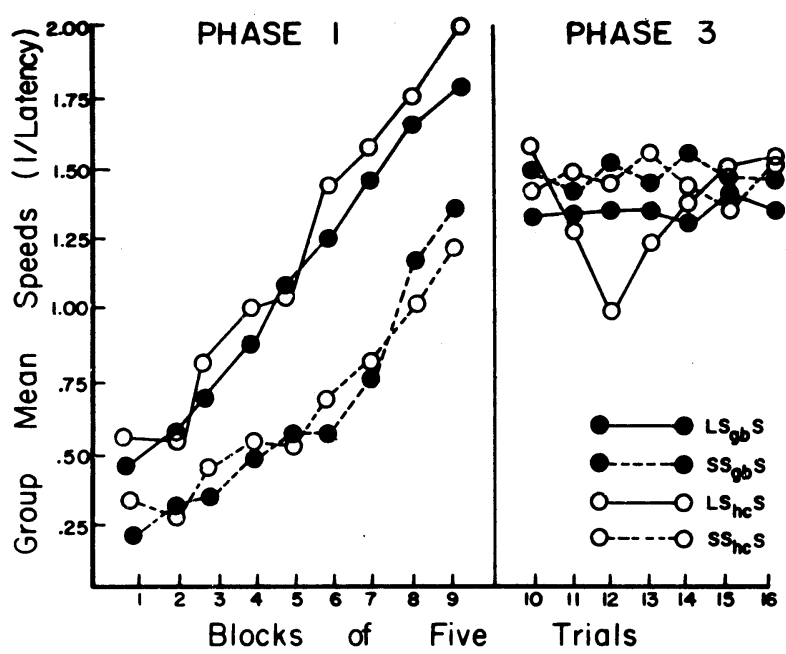

Figure 1. Group mean speeds as a function of blocks of five trials for subjects receiving placement trials in the goalbox $\left(\mathrm{LS}_{\mathrm{gb}} \mathrm{S}\right.$ and $\left.\mathrm{SS}_{\mathrm{gb}} \mathrm{S}\right)$ or in a housing cage $\left(\mathrm{LS}_{\mathrm{hc}} \mathrm{S}\right.$ and $\left.\mathrm{SS}_{\mathrm{hc}} \mathrm{S}\right)$.

(Group $\mathrm{SS}_{\mathrm{hc}} \mathrm{S}$ ) at Block 12 (NCE). However, the NCE dissipated over time.

A treatment (large and small reward during Phase 1) by location (home cage vs. goalbox) analysis of variance was performed on the data from Block 12 . The analysis yielded a significant Location by Treatment interaction $[\mathrm{F}(1,28)=6.21, \mathrm{p}<.01]$. Tukey (a) paired comparisons between group mean running speeds from Block 12 showed that Group $\mathrm{LS}_{\mathrm{hc}} \mathrm{S}$ ran significantly slower than Group $\mathrm{SS}_{\mathrm{hc}} \mathrm{S}(\mathrm{p}<.01)$. However, a reliable NCE was not shown by Groups $\mathrm{LS}_{\mathrm{gb}} \mathrm{S}$ and $\mathrm{SS}_{\mathrm{gb}} \mathrm{S}(\mathrm{p}<.05)$. It should also be mentioned that at Block 14, none of the comparisons approached significance $(p>.10)$.

\section{DISCUSSION}

The finding of transient depressed speeds early in the postshift period following a shift to small magnitude of reward is consistent with the results of previous studies (e.g., Capaldi \& Lynch, 1967; Crespi, 1942; Ehrenfreund, 1971; Gonzales et al., 1962). More important, however, was the finding that a shift from contingent large to noncontingent small to contingent small magnitude of reward produced a NCE when the noncontingent small-reward condition (Phase 2) was presented in a gray home cage rather than in a gray goalbox with most of the stimulus properties (brightness, texture, size, shape, and cover) of the gray alley. The latter findings imply that alley cues other than brightness played the dominant role in mediating a NCE. If brightness were the dominant cue in the present study, then neither placement group should have shown a NCE, since both groups received, in Stage 2, the same brightness (gray) that was present in Stage 1. The fact that Group $\mathrm{LS}_{\mathrm{hc}} \mathrm{S}$ showed a NCE during Stage 3 implies that frustration did not develop and dissipate for this group during Stage 2, since the probable dominant cue or cues (texture, size, shape, and cover) eliciting anticipation for large reward in Stage 2 were not present during this period. However, the fact that Group $\mathrm{LS}_{\mathrm{gb}} \mathrm{S}$ did not show a
NCE implies that frustration did develop and dissipate for this group during Stage 2, since the probable dominant cue or cues were present during this period.

The present findings also suggest that Capaldi's (1978) findings that a NCE occurred with a brightness change during postshift training may not be in conflict with a frustration account of the "depression" effect. Rather, the present results indicate that cues other than brightness may have been mediating the NCE in the Capaldi study by eliciting anticipation of large reward during the postshift phase, since these cues were present during this period. It should be mentioned that the present study presented no information as to which cue of the stimulus complex (texture, shape, size, or cover) or interactions therein may have mediated the NCE in the present or in Capaldi's research. Further research should factorize these cue variables to investigate what effect they may have on the NCE.

\section{REFERENCES}

Amsel, A. The role of frustrative nonreward in noncontinuous reward. Psychological Bulletin, 1958, 95, 102-119.

Amsel, A. Frustrative nonreward in partial reinforcement and discrimination learning: Some recent history and theoretical extensions. Psychological Review, 1962, 69, 306-328.

BLACK, R. W. Shifts in magnitude of reward and contrast effects in instrumental and selective learning: A reinterpretation. Psychological Review, 1968, 75, 114-126.

CALEF, R. S. The effect of large and small magnitude of intertrial reinforcement on successive contrast effects. Psychonomic Science, 1972, 29, 309-312.

Calef, R. S., Calef, R. A., Prochaska, A. D., \& Geller, E. S. Negative contrast as a function of reinforcement location and consistent vs. varied reward magnitude. Bulletin of the Psychonomic Society, 1978, 12, 471-474.

Calef, R. S., Hopkins, D. C., McHewitt, E. R., \& Maxwell, F. R. Performance to varied reward following continuous reward training in the runway. Bulletin of the Psychonomic Society, 1973, 2, 103-104.

Capaldi, E. D. Effects of changing alley color on the successive negative contrast effect. Bulletin of the Psychonomic Society, 1978, 12, 69-70.

Capaldi, E. J., \& Lynch, D. Repeated shifts in reward magnitude: Evidence in favor of an associational and absolute (noncontextual) interpretation. Journal of Experimental Psychology, $1967,75,226-235$.

Crespi, L. P. Quantitative variation in incentive and performance in the white rat. American Journal of Psychology, 1942, 55, 467-517.

EhrenfreUnd, D. Effect of drive on successive magnitude shift in rats. Journal of Comparative and Physiological Psychology, 1971, 76, 418-423.

Gonzales, R. C., Gleitman, H., \& Bitterman, M. E. Some observations on the depression effect. Journal of Comparative and Physiological Psychology, 1962, 55, 578-581.

Ludvigson, H. W., \& GAY, S. Differential reward conditioning: $\mathrm{S}^{-}$contrast as a function of the magnitude of $\mathrm{S}^{+}$. Psychonomic Science, 1966, 5, 289-290.

Maxwell, F. R., Calef, R. S., Murray, D. W., Shepard, J. C., \& Norville, R. A. Positive and negative successive contrast effects following multiple shifts in reward magnitude under high drive and immediate reinforcement. Animal Learning \& Behavior, 1976, 4, 480-484.

(Received for publication January 7, 1980.) 